Revista de Matemática: TeOría y APliCACiOnes 2015 22(2) : 311-323

CIMPA - UCR ISSN: 1409-2433 (PRINT), 2215-3373 (ONLINE)

\title{
COLORACIÓN DE GRÁFICAS SUAVES
}

\section{SOFT GRAPH COLORING}

\author{
PedRo LaRa-VelázQuez \\ Miguel ANGel GutiéRREZ-ANDRADE ${ }^{\dagger}$ \\ SERGIO G. DE-LOS-COBOS-SILVA \\ ERIC ALFREDO RINCÓN-GARCÍA ${ }^{\S}$
}

Received: 25/Feb/2014; Revised: 12/Mar/2015;

Accepted: 19/May/2015

\footnotetext{
*Universidad Autónoma Metropolitana-Iztapalapa, Departamento de Ingeniería Eléctrica, Av. San Rafael Atlixco 186, Col. Vicentina, Del. Iztapalapa, México D.F., C.P. 09340, México. EMail: plara@xanum.uam.mx

${ }^{\dagger}$ Misma dirección que/same address as: P. Lara. E-Mail: gamma@xanum.uam.mx

${ }^{\ddagger}$ Misma dirección que/same address as: P. Lara. E-Mail: cobos@ @anum.uam.mx

${ }^{\S}$ Universidad Autónoma Metropolitana-Azcapotzalco, Departamento de Sistemas. Av. San Pablo 180 Col. Reynosa Tamaulipas CP 02200, México D.F., México E-Mail: rigaeral@correo.azc.uam.mx
} 


\title{
Resumen
}

En este trabajo se propone un modelo de Coloración en Gráficas Suaves donde se colorea con base en ponderaciones sobre las aristas de la gráfica. Se muestra que este modelo es muy flexible e incluye otros problemas similares, tales como los problemas de Coloración Mínima, Coloración Equitativa, Coloración de Gráficas Débiles y Coloración Robusta. Se proponen también un modelo binario lineal de solución y algunas instancias de prueba.

Palabras clave: coloración de graficas suaves; teoría de graficas; coloración sobre vértices.

\begin{abstract}
In this paper a Soft Graph Coloring Model is proposed, which is colored based on weights on the edges of the graph. It is shown that this model is very flexible and includes other similar problems such as Minimal, Equitable, Weak, and Robust Graph Coloring. A linear binary solution model and some test instances are also proposed.
\end{abstract}

Keywords: soft graph coloring; graph theory; coloring on vertexes.

Mathematics Subject Classification: 90C90, 90C10, 05C15.

\section{Introducción}

La coloración de gráficas suaves es una generalización del problema de coloración [6] en donde dada una gráfica con ponderaciones en las aristas, buscamos una coloración que minimice la suma de las aristas con el mismo color en ambos extremos. Por ser una generalización del problema de coloración robusta, se sabe que este problema es del tipo NP-duro y se necesita utilizar metaheurísticas en instancias mayores a 20 vértices.

En los últimos años se ha trabajado extensamente en problemas de coloración, especialmente en problemas de calendarización [2], [8], [10] y [7], los cuales suelen estar fragmentados en varios modelos y a pesar de algunos esfuerzos por hacer modelos integradores [4], [11] y [12]. Estos suelen ser inclusivos como modificaciones del problema que ellos tratan, pero no profundizan en encontrar un modelo que incluya propiedades comunes a todos los problemas de gráficas y que aporte información adicional al problema.

Este trabajo se distribuye de la siguiente forma: el problema de coloración de gráficas suaves es descrito en la sección 2, así la dureza, solidez y resiliencia, 
que son propiedades de cada coloración. En la sección 3 se propone un modelo binario que resuelve este problema exactamente. La sección 4 muestra que este problema engloba el problema de coloración mínima, el problema de coloración equitativa, el problema de coloración robusta y el problema de coloración débil. En la sección 5 se proponen algunas instancias aleatorias con diferentes distribuciones estadísticas de los pesos en las aristas. En la sección 6 se muestran los resultados obtenidos clasificados pro tipo de distribución. Finalmente se presentan las conclusiones y trabajo subsecuente.

\section{Definición del problema}

La Coloración de Gráficas Suaves (CGS) es un caso especial del problema de coloración de gráficas donde se busca una coloración que minimice la dureza de la coloración, es decir, se busca una coloración que minimice la suma de las penalizaciones entre las aristas incidentes cuyos vértices tienen el mismo color. El problema se puede definir como sigue: Sea $G=(V, E)$ una gráfica completa no dirigida, es decir, el conjunto de los vértices $V=\{1,2, \ldots, n\}$ en $G$ y todas sus aristas posibles $(i, j)$, donde:

$$
G=(V, E) ;|V|=n ;|E|=n(n-1) / 2 .
$$

Se define una penalización en cada arista $(i, j)$, denotada por $p_{i j}$ tal que:

$$
p_{i j} \geq 0, \quad \forall(i, j) \in E .
$$

Una función de coloración en los vértices de $G=(V, E)$ se define como:

$$
C^{k}: 1,2, \ldots, n \rightarrow 1,2, \ldots, k
$$

donde $k$ es el número total de colores $1<k<n$ que identifica $C(i)$ como el color en el vértice $i$. Para una coloración $C^{k}$ en una gráfica la función de dureza de la coloración $C^{k}$ está dada por:

$$
H\left(C^{k}\right)=\sum_{(i, j) \in E,} P_{C^{k}(i)=C^{k}(j)} p_{i j} .
$$

El objetivo del problema de CGS es encontrar la coloración $C_{o p}^{k}$ que minimiza la dureza $H\left(C_{o p}^{k}\right)$. 


\subsection{Solidez y resiliencia de una coloración}

Dada una coloración con $k$ colores en una gráfica completa con $n$ vértices, el número promedio $m$ de vértices pintados con el mismo color es $m=n / k$ y el número de aristas compartidas por $m$ vértices son las combinaciones $\mathrm{C}(m, 2)=$ $m(m-1) / 2$ por lo que el número promedio de penalizaciones incluidas en la función de dureza debe ser proporcional al número promedio de vértices pintados con el mismo color multiplicado por el número de colores. Definimos la función de solidez de una coloración como la dureza de un gráfico dividido por el número medio de aristas que contribuyen a la dureza:

$$
S\left(C_{o p}^{k}\right)=\frac{H\left(C_{o p}^{k}\right)}{k m(m-1) / 2}=\frac{2 H\left(C_{o p}^{k}\right)}{k \frac{n}{k}\left(\frac{n}{k}-1\right)}=\frac{2 k H\left(C_{o p}^{k}\right)}{n(n-k)} .
$$

La resiliencia de una coloración $C^{k}$ se define como el porcentaje en que disminuye la solidez de una coloración con $k-1$ colores con respecto a la realizada con $k$ de ellos y se puede expresar como sigue:

$$
R\left(C_{o p}^{k}\right)=\frac{S\left(C_{o p}^{k-1}\right)-S\left(C_{o p}^{k}\right)}{S\left(C_{o p}^{k}\right)} .
$$

Si resulta que al añadir un color adicional a la coloración de la gráfica, hay un aumento significativo en su resiliencia, eso significa que hemos encontrado un número adecuado de clases para clasificar.

Finalmente si obtenemos la resiliencia para todas las coloraciones consecutivas desde 1 hasta $n$, y si pensamos en cada color como un grupo para clasificar a los vértices (objetos), los valores más grandes mostrarán las mejores opciones de número de clases que se pueden utilizar para clasificar el conjunto.

\section{Modelo binario entero}

Dada una gráfica no dirigida $G=(V, E)$ con $|V|=n$ y $|E|=n(n-1) / 2$ se desea obtener una coloración $C_{o p}^{k}$ usando $k$ colores. Para resolver este problema definimos el modelo de programación binaria siguiente:

$$
\min z=\sum_{(i, j) \in E} p_{i j} y_{i j}
$$

sujeto a:

$$
\sum_{l=1}^{k} x_{i l}=1 \forall i \in\{1, \ldots, n\}
$$




$$
\begin{gathered}
x_{i l}+x_{j l}-1 \leq y_{i j} \forall(i, j) \in E \text { y } \forall l \in\{1, \ldots, k\} \\
x_{i l} \in\{0,1\} \forall i \in E \text { y } \forall l \in\{1, \ldots, k\} \\
y_{i} \in\{0,1\} \quad \forall(i, j) \in E .
\end{gathered}
$$

donde:

$$
\begin{gathered}
x_{i l}=\left\{\begin{array}{ll}
1 & \text { si } C(i)=l \forall i \in\{1, \ldots, n\} \text { y } \forall l \in\{1, \ldots, k\} \\
0 & \text { en otro caso }
\end{array},\right. \\
y_{i j}= \begin{cases}1 & \text { si para alguna } l \in\{1, \ldots, k\} \text { se cumple que } x_{i l}=x_{j l}=1 \\
0 & \text { en otro caso. }\end{cases}
\end{gathered}
$$

El conjunto de ecuaciones del sistema (7) garantiza que cada vértice tiene asignado solamente un color; el conjunto de desigualdades hace que la variable $y_{i j}$ valga 1 si se colorean los vértices $i$ y $j$ con el mismo color y cero si los vértices $i$ y $j$ tienen colores diferentes. En el primer caso se activa la penalización $p_{i j}$ en la función objetivo $z$. La solución al problema (7) obtiene la coloración mínima $C_{o p}^{k}$ tomando $C_{o p}^{k}(i)=l$ si $x_{i l}=1$, donde $x_{i l} \forall i \in\{1, \ldots, n\}$ y $\forall l \in\{1, \ldots, k\}$, son los valores de las variables de decisión obtenidos en la solución óptima del problema (7) y el valor mínimo $z^{*}$ de la función objetivo es igual a $H\left(C_{o p}^{k}\right)$.

El sistema (7) tiene $n k$ variables $x_{i l}$ y $n(n-1) / 2$ variables $y_{i j}$ por lo que el número total de variables binarias en el modelo 1 es $n k+n(n-1) / 2$. Por otro lado el número de ecuaciones es igual a $n$ y el número de desigualdades es igual a $k n(n-1) / 2$, por lo tanto, el número total de restricciones es igual a: $n+k n(n-1) / 2$.

\section{Otros modelos incluidos en CGS}

a) Coloración mínima [6]. En este problema se tiene una gráfica no dirigida en la cual cuando se tiene una arista que conecta a dos vértices, dichos vértices no pueden pintarse con el mismo color. Este modelo se puede plantear con la siguiente función objetivo:

$$
\min z=\sum_{(i, j) \in E} p_{i j} y_{i j},
$$

donde $A=\left(p_{i j}\right)$ es la matriz de adyacencia que cumple para cada entrada uno de dos valores, $p_{i j}=1$ si los vértices $i, j$ están conectados y $p_{i j}=0$ si no lo están. Para una coloración con el número mínimo de colores que de una dureza cero, es una coloración con el número cromático. 
b) Coloración equitativa [9]. En este modelo se busca colorear una gráfica con clases de color que discrepan a lo más en un elemento. La función objetivo se puede formular de la siguiente forma:

$$
\min z=\sum_{(i, j) \in E} p_{i j} y_{i j}
$$

donde $p_{i j}=1$ si no pertenece a la matriz de adyacencia y vale $1+\varepsilon$, por ejemplo, $p_{i j}=1.00001$ si sí pertenece. El valor de ponderación tan cercano en ambos conjuntos garantiza que el número de colores está equitativamente distribuido, porque en una gráfica completa, al pintar 1 aristas de un mismo color la aportación a la dureza de ese color es $l(l-1) / 2$, a medida de que aumente $l$, aumenta la rigidez, por lo que cada color tenderá a pintar aproximadamente $n / k$ que es un valor cercano a $l$. Si no se puede garantizar una coloración equitativa, por ejemplo, en una gráfica donde $n$ vértices están conectados a un único vértice, el valor de dureza será un número fraccional. La factibilidad de la solución se garantiza cuando el valor de dureza es un número entero.

c) Coloración de gráficas débiles [7]. En este modelo, se colorea una gráfica con menos colores que el número cromático. El objetivo es crear una gráfica con el mínimo número de incompatibilidades. Visto desde otra perspectiva, es el modelo de coloración mínima cuando se tienen menos colores que el número cromático, ya que en este caso $p_{i j}=1$ es un vértice que está mal coloreado. Dentro del esquema de coloración de gráficas suaves, se busca minimizar la función de dureza que incluye todas las $p_{i j}=1$, cuyos extremos están pintados con el mismo color. La función de dureza da exactamente el número de incompatibilidades en la coloración de la gráfica.

d) Coloración robusta [14], [11]. Este problema contiene dos gráficas, la primera del tipo coloración mínima, denominada $G=(V, E)$, la cual es una restricción del problema. Adicionalmente se tiene una gráfica en las aristas complementarias penalizadas con valor $v_{i j}$, denominada $\bar{E}$, con valores reales en un intervalo $(0,1)$. En este problema el objetivo es minimizar la suma de los valores de las aristas penalizadas en $\bar{E}$, manteniendo válidas las incompatibilidades en $E$. Este problema tiene la propiedad de que las aristas de ambas gráficas son mutuamente excluyentes. Para convertir este problema a un modelo en gráficas suaves, dada la función objetivo:

$$
\min z=\sum_{(i, j) \in E} p_{i j} y_{i j}
$$

Se re-penalizan las aristas de la gráfica $G=(V, E)$ de la siguiente manera:

$$
\begin{array}{lll}
n^{2} & \text { si } & i, j \in E \\
v_{i j} & \text { si } & i, j \in \bar{E} .
\end{array}
$$


Penalizando las aristas de la gráfica original con un valor igual a $n^{2}$ se garantiza que al encontrar una coloración con una dureza menor que $n^{2}$, se tiene una coloración válida mínima: Esta gráfica es válida en el problema de coloración mínima porque se han excluido todas las aristas de la gráfica $E$, y dicha solución tiene un costo menor a $n^{2}$ ya que todas las penalizaciones son positivas y estrictamente menores que uno. Para la gráfica complementaria se conservan las penalizaciones originales. El número cromático es aquel número de colores donde se obtenga por primera vez una coloración menor que $n^{2}$. Para más colores que el número cromático, cualquier coloración óptima de gráficas suaves es una coloración óptima del problema de coloración robusta con esa cantidad específica de colores.

\section{Instancias utilizadas}

Por lo mencionado en la sección 2, además de la información encontrada en la función de dureza, que es la función objetivo, se puede saber cuál es el valor medio de ponderaciones mediante la función de solidez, y, dado que se puede encontrar todas las coloraciones posibles, la función de resiliencia es un indicador de la cantidad óptima de colores para pintar dicha gráfica. Por las razones antes mencionadas, en este trabajo se propusieron instancias que hacen uso de las propiedades de dureza, rigidez y resiliencia y su comportamiento para diferentes distribuciones de ponderaciones.

Un punto a considerar en este problema es la densidad de la gráfica, que es una característica considerada en todas las coloraciones que se modelan mediante gráficas suaves. En coloración robusta se considera además la gráfica complementaria y en todas ellas, la frecuencia de ocurrencia de cada ponderación es uniforme. Aunque las gráficas aleatorias uniformes son difíciles de colorear [14] y [13]. Considerando únicamente una distribución uniforme de ponderaciones en las aristas no se puede obtener información sobre el número de colores óptimo a utilizar en el problema.

Las matrices de ponderación que se utilizan en los problemas de coloración mínima, equitativa y débil son matrices binarias, y las matrices del problema de coloración robusta es una mezcla de matriz binaria con distribución uniforme en el intervalo $(0,1)$. Estas matrices no dan mucha información sobre el modelo así que se consideraron instancias tipo coloración robusta, sin perder generalidad, que se encuentran con diferentes distribuciones en el intervalo $(0,1)$, aunque, en principio, para coloración de gráficas suaves se puede considerar cualquier número real en las ponderaciones. 
Se generaron 10 instancias para 10 y 15 vértices. Aunque en coloración de gráficas suaves se puede utilizar, en principio, cualquier número real, para las instancias propuestas se consideró el intervalo continuo $(0,1)$. Cada una con tres diferentes distribuciones de ponderaciones: uniforme, triangular simétrica y triangular con moda en cero. Se resolvió el problema para cada instancia incluyendo dureza, solidez y resiliencia. El nombre de la instancia determina cuál es su distribución de penalizaciones en las aristas, por ejemplo, las instancias TRI15, UNI10 y TRIC15 se refieren a instancias aleatorias triangulares simétricas con 15 vértices, uniforme con 10 vértices y triangular con moda en cero con 15 vértices respectivamente.

\section{Resultados}

A continuación se muestran tres tablas sumario de las instancias propuestas. En cada tabla se incluye el número de colores utilizado para pintar la gráfica, así como la dureza, solidez y resiliencia promedio. El valor mostrado es la media de 10 instancias generadas aleatorias con las distribuciones indicadas.

\begin{tabular}{c|ccc}
\hline \hline UNI10 & Dureza Prom & Solidez & Resiliencia \\
\hline 1 & 22.47478 & 0.49943956 & \\
2 & 7.35431 & 0.36771550 & 0.358222744 \\
3 & 3.08946 & 0.26481086 & 0.388596767 \\
4 & 1.27464 & 0.16995200 & 0.558150873 \\
5 & 0.66839 & 0.13367800 & 0.271353551 \\
6 & 0.32526 & 0.09757800 & 0.369960442 \\
7 & 0.13999 & 0.06532867 & 0.493647505 \\
8 & 0.05962 & 0.04769600 & 0.369688583 \\
9 & 0.01724 & 0.03103200 & 0.536994071 \\
10 & 0.00000 & & \\
\hline \hline
\end{tabular}

Tabla 1: Instancia con distribución uniforme 10 vértices.

En ambos casos podemos encontrar un pequeño incremento en la resiliencia en la parte central de la distribución, así como en la parte final. Cabe mencionar que el incremento de resiliencia en la parte final de la gráfica se puede deber a 


\begin{tabular}{c|ccc}
\hline \hline UNI15 & Dureza Prom & Solidez & Resiliencia \\
\hline 1 & 54.39909 & 0.518086571 & \\
2 & 20.27276 & 0.415851487 & 0.245845181 \\
3 & 10.23464 & 0.341154667 & 0.21895295 \\
4 & 5.66637 & 0.274733091 & 0.241767657 \\
5 & 3.2759 & 0.218393333 & 0.257973798 \\
6 & 1.89399 & 0.168354667 & 0.297221738 \\
7 & 1.04433 & 0.1218385 & 0.381785451 \\
8 & 0.6345 & 0.096685714 & 0.26014997 \\
9 & 0.4127 & 0.08254 & 0.17138011 \\
10 & 0.27428 & 0.073141333 & 0.128500073 \\
11 & 0.1716 & 0.06292 & 0.162449672 \\
12 & 0.09202 & 0.049077333 & 0.282058248 \\
13 & 0.04171 & 0.036148667 & 0.357652657 \\
14 & 0.01547 & 0.028877333 & 0.25180072 \\
15 & 0.00000 & & \\
\hline \hline
\end{tabular}

Tabla 2: Instancia con distribución uniforme 15 vértices.

que las últimas aristas tienen valores de ponderación muy pequeños, por lo que es muy probable que se comporte como un evento poissoniano.

Aunque en esta distribución se presenta el mismo fenómeno que en el conjunto de instancias anterior, cabe resaltar que el valor de resiliencia disminuye a casi la mitad aunque los valores de dureza y solidez se mantienen esencialmente iguales. De cualquier forma, la resiliencia tiene nuevamente un máximo aproximadamente a la mitad, y, posiblemente por el fenómeno antes mencionado, otro máximo en la parte final. 


\begin{tabular}{c|ccc}
\hline \hline TRI10 & Dureza Prom & Solidez & Resiliencia \\
\hline 1 & 21.91829 & 0.48707311 & \\
2 & 7.92131 & 0.39606550 & 0.229779193 \\
3 & 4.01867 & 0.34445743 & 0.149824237 \\
4 & 2.25912 & 0.30121600 & 0.143556214 \\
5 & 1.29213 & 0.25842600 & 0.165579315 \\
6 & 0.79388 & 0.23816400 & 0.08507583 \\
7 & 0.48263 & 0.22522733 & 0.057438262 \\
8 & 0.25127 & 0.20101600 & 0.120444807 \\
9 & 0.10505 & 0.18909000 & 0.063070496 \\
10 & 0.00000 & & \\
\hline \hline
\end{tabular}

Tabla 3: Instancia con distribución triangular simétrica 10 vértices.

\begin{tabular}{c|ccc}
\hline \hline TRI15 & Dureza Prom & Solidez & Resiliencia \\
\hline 1 & 51.96656 & 0.494919619 & \\
2 & 20.63456 & 0.423273026 & 0.169268035 \\
3 & 11.07321 & 0.369107 & 0.146748844 \\
4 & 6.63829 & 0.321856485 & 0.146806162 \\
5 & 4.172 & 0.278133333 & 0.157202127 \\
6 & 2.84592 & 0.252970667 & 0.099468713 \\
7 & 1.85566 & 0.216493667 & 0.168489917 \\
8 & 1.27782 & 0.194715429 & 0.111846494 \\
9 & 0.93195 & 0.18639 & 0.044666713 \\
10 & 0.67106 & 0.178949333 & 0.04157974 \\
11 & 0.46269 & 0.169653 & 0.054796162 \\
12 & 0.29309 & 0.156314667 & 0.085330018 \\
13 & 0.15306 & 0.132652 & 0.17838153 \\
14 & 0.05059 & 0.094434667 & 0.404696016 \\
15 & 0.00000 & & \\
\hline \hline
\end{tabular}

Tabla 4: Instancia con distribución triangular simétrica 15 vértices. 


\begin{tabular}{c|ccc}
\hline \hline TRIC10 & Dureza Prom & Solidez & Resiliencia \\
\hline 1 & 15.17117 & 0.33713711 & \\
2 & 4.80163 & 0.24008150 & 0.404261099 \\
3 & 2.01027 & 0.17230886 & 0.393320715 \\
4 & 0.91903 & 0.12253733 & 0.406174367 \\
5 & 0.4334 & 0.08668000 & 0.413674819 \\
6 & 0.19333 & 0.05799900 & 0.494508526 \\
7 & 0.1089 & 0.05082000 & 0.141263282 \\
8 & 0.05161 & 0.04128800 & 0.230866111 \\
9 & 0.01947 & 0.03504600 & 0.178108771 \\
10 & 0.00000 & & \\
\hline \hline
\end{tabular}

Tabla 5: Instancia con distribución triangular con moda en cero, 10 vértices.

\begin{tabular}{c|ccc}
\hline \hline TRIC15 & Dureza Prom & Solidez & Resiliencia \\
\hline 1 & 35.43438 & 0.337470286 & \\
2 & 12.17839 & 0.249813128 & 0.350890916 \\
3 & 5.88542 & 0.196180667 & 0.273383012 \\
4 & 3.03702 & 0.147249455 & 0.332301483 \\
5 & 1.64458 & 0.109638667 & 0.343043098 \\
6 & 0.88569 & 0.078728 & 0.392626088 \\
7 & 0.50346 & 0.058737 & 0.340347651 \\
8 & 0.28536 & 0.043483429 & 0.350790449 \\
9 & 0.18911 & 0.037822 & 0.149686124 \\
10 & 0.11958 & 0.031888 & 0.186088811 \\
11 & 0.07318 & 0.026832667 & 0.188402196 \\
12 & 0.04062 & 0.021664 & 0.23858321 \\
13 & 0.01981 & 0.017168667 & 0.261833573 \\
14 & 0.00573 & 0.010696 & 0.605148342 \\
15 & 0.00000 & & \\
\hline \hline
\end{tabular}

Tabla 6: Instancia con distribución triangular con moda en cero, 15 vértices. 
Lo más evidente que se puede observar en esta distribución es que la dureza y la solidez disminuyeron en aproximadamente un 50\%, esto se debe a que en las primeras distribuciones la media de penalizaciones se encontraba cerca de 0.5 (lo que se puede observar en la solidez cuando se pinta con un solo color, como todas las aristas están incluidas y es una gráfica completa, la solidez da el valor promedio de las penalizaciones de la gráfica), en las distribuciones uniforme y triangular simétrica, dicha media es cercana a $0.5 \mathrm{y}$, en el caso de la triangular con moda en cero, la media es cercana a $1 / 3$.

Otra característica notable de este conjunto de instancias es que la resiliencia aumentó considerablemente, siendo de las tres gráficas las de valores mayores. En este conjunto, la resiliencia se mantiene alta con pocos colores hasta llegar a la mitad. Nuevamente, se observa un incremento en la parte final.

\section{Conclusiones}

En los tres conjuntos de instancias se observa que la resiliencia, que es el parámetro que nos permite encontrar una cantidad adecuada de colores, tiene valores altos para pocos colores. Esto es algo afortunado ya que el principal uso del problema de coloración es la asignación a usuarios de recursos escasos. Como una regla general, dados problemas con distribuciones similares a las presentadas (que son, prácticamente, cualquier problema), es posible encontrar asignaciones con la mitad o menos de recursos que usuarios. Por otra parte, tenemos un incremento de resiliencia significativo cuando el número de usuarios y de colores casi son iguales, lo cual puede ser considerado para problemas donde casi todos los usuarios tienen un recurso propio, pero por algún evento extraordinario, dos de ellos tienen que compartir el recurso.

\section{Agradecimientos}

Los autores desean agradecer a los árbitros anónimos por sus valiosas sugerencias.

\section{Referencias}

[1] Burke, E.K.; Jackson, K.; Kingston, J.; Weare, R. (1997) "Automated university timetabling: the state of the art", Comput. J. 40: 565-571.

[2] Burke, E.K.; Petrovic, S. (2002) "Recent research directions in automated timetabling”, Eur. J. Opl. Res. 140: 266-280. 
[3] Cangalovic, M.; Schreuder, J.A.M. (1991) "Exact colouring algorithm for weighted graphs applied to timetabling problems with lectures of different lengths", Eur. J. Opl. Res. 51: 248-258.

[4] Carter, M.W.; Laporte, G. (1998) "Recent developments in practical course timetabling", in: E.R. Burke \& M. Carter (Eds) Practice and Theory of Automated Timetabling II, Second International Conference, PATAT'97, Toronto, Canada: 3-19.

[5] Cowen, L.; Goddard, W.; Jesurum C.E. (1997) "Defective Coloring Revisited", J. Graph. Theory 24(3): 205-219.

[6] Diestel, R. (2000) Graph Theory. Springer-Verlag, New York.

[7] Kuhn, F. (2009) "Weak graph colorings: distributed algorithms and applications", SPAA'09, Calgary, Alberta, Canada: 138-144.

[8] McCollum, B.; Schaerf, A.; Paechter, B.; McMullan, P.; Lewis, R.; Parkes, A.; Di Gaspero, L.; Qu, R.; Burke E. (2009) "Setting the research agenda in automated timetabling: the Second International Timetabling Competition", INFORMS Journal on Computing, doi: 10.1287/ijoc.1090.0320.

[9] Meyer, W. (1973) "Equitable coloring", American Mathematical Monthly (Mathematical Association of America) 80: 920-922, doi: $10.2307 / 2319405$

[10] Nandhini, M.; Kanmani, S. (2009) "A survey of simulated annealing methodology for university course timetabling", International Journal of Recent Trends in Engineering 1: 255-257.

[11] Ramírez, J. (2001) Extensiones del problema de coloración de gráfos. Tesis Doctoral, Universidad Complutense de Madrid, Madrid, España.

[12] Roberts, F.S. (1991) “T-colorings of graphs: recent results and open problems $\hat{A}^{\prime} \hat{A}^{\prime}$, Discrete Math. 93: 229-245.

[13] Wang, F.; Xu, Z. (2013) "Heuristics for robust graph coloring", J. Heuristics 19: 529-548. doi: 10.1007/s10732-011-9180-4

[14] Yañez, J.; Ramírez, J. (2003) “The robust coloring problem”, European Journal of Operational Research, 148(3): 546-558. 
